\title{
COHEN-MACAULAY ALGEBRAIC MONOIDS
}

\author{
LEX E. RENNER
}

\begin{abstract}
Let $E$ be an irreducible, normal, algebraic monoid with group of units $\left(j=\mathrm{Gl}_{2}(K), \mathrm{Sl}_{2}(K) \times K^{*}\right.$ or $\mathrm{P} \mathrm{Hl}_{2}(K) \times K^{*}$. Then $E$ is a Cohen-Macaulay algebraic varicty.
\end{abstract}

0. Introduction. An algebraic monoid $E$ is an algebraic variety together with an associative morphism $m: E \times E \rightarrow E$ and a two-sided unit $1 \in E$ for $m$.

Several authors $[6,8]$ are currently studying the theory of algebraic monoids. Prior to this, the first major geometric breakthrough [2] was published in 1972. In that work, Hochster proved that any normal $D$-monoid is a Cohen-Macaulay algebraic variety. The extent to which that result can be generalized to more general monoids is not known; this work is an optimistic addition.

Let $X$ be an irreducible algebraic variety. $X$ is Cohen-Macaulay if for all $x \in X$ there exists a regular sequence $\left\{f_{1}, \ldots, f_{m}\right\} \subseteq \mathfrak{c}_{x, X}$, which is a system of parameters.

THEOREM. Suppose E is an irreducible, normal, algebraic monoid with group of units $\mathrm{Gl}_{2}(K), \mathrm{PGl}_{2}(K) \times K^{*}$ or $\mathrm{Sl}_{2}(K) \times K^{*}$. Then $E$ is a Cohen-Macaulay algebraic variety.

There are two main points to the proof.

(1) In [11] it is proved that any finite, normal, separable, abelian covering of a smooth variety is Cohen-Macaulay. That result is generalized here.

(2) Given any monoid $E$, as in the theorem, there exists a diagram

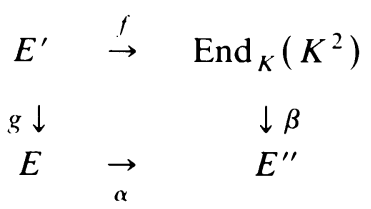

such that all morphisms are finite and dominant and have $D$-group kernels. (1) above is then applied to the morphism $f$ to yield the result.

1. Notation and terminology. Let $K$ be an algebraically closed field. An algebraic monoid is an affine algebraic variety, defined over $K$, together with an associative morphism $m: E \times E \rightarrow E$ and a two-sided unit $l \in E$ for $m$. Our general reference here is [6].

Received by the editors July 12, 1982 and, in revised form, April 7, 1983.

1980 Mathematics Subject Classification. Primary 20M99: Secondary 14M05.

Key words and phrases. Algebraic monoid, Cohen-Macaulay, D-group. 
If $E$ is an algebraic monoid then $G(E)=\left\{x \in E \mid x^{-1} \in E\right\}$ is an affine algebraic group and $G(E) \subseteq E$ is an open subset in the Zariski topology. $E^{\circ}$, the irreducible component of 1 , is the unique maximal closed, irreducible submonoid of $E$.

An affine variety is completely determined by its $K$-algebra of regular functions. So, an affine, algebraic monoid $E$ is completely determined by morphisms $\Delta$ : $K[E] \rightarrow K[E] \otimes K[E]$ and $\varepsilon: K[E] \rightarrow K$ such that

$$
(\Delta \otimes 1) \circ \Delta=(1 \otimes \Delta) \circ \Delta \text { and }(\eta \circ \varepsilon, 1) \circ \Delta=(1, \eta \circ \varepsilon) \circ \Delta=1 \text {, }
$$

where $\eta: K \rightarrow K[E]$ is the unit of the $K$-algebra structure of $K[E] . K[E]$ is thus a $K$-bigebra. The functor $E \rightarrow K[E]$ is an equivalence between the category of algebraic monoids and the category of finitely generated $K$-bigebras.

Let $Z$ be an irreducible, algebraic monoid. $Z$ is a $D$-monoid if $G(Z)$ is a torus. $X(Z)=\operatorname{Hom}(Z, K)$ is the set of characters of $Z$. If $E$ is any irreducible algebraic monoid, a maximal $D$-submonoid $Z \subseteq E$ is the closure in $E$ of a maximal torus $T$ of $G(E) . Z$ is determined to within an isomorphism by the finitely generated, commutative monoid $X(Z)$.

An irreducible, algebraic monoid $E$ is reductive if $G(E)$ is a reductive algebraic group.

If $e=e^{2} \in E$ let $G(e)$ denote the algebraic group of units of the algebraic monoid $e E e$.

$I(E)=\left\{e \in E \mid e^{2}=e\right\}$.

$E$ is regular if for all $x \in E$ there exists $g \in G(E)$ and $e \in I(E)$, such that $x=g e$.

For a survey of many of the known results on algebraic monoids the reader should consult [6]. A systematic treatment of some of the fundamental topics has been initiated in [8].

2. Finite $D$-group actions. To establish the main results of the paper, I have been led to consider certain morphisms $f: G \rightarrow H$ such that $\operatorname{kernel}(f)$ is a (not necessarily reduced) finite $D$-group scheme. The purpose of this section is to generalize the results of $\mathrm{P}$. Roberts [11] that can be applied to these morphisms $f$ above. Our reference for the theory of $D$-groups is $[13,2.2]$. The subtlety arises only if the characteristic of the ground field divides the order of the group scheme.

Definition. Let $G$ be an affine group scheme over $K$. $G$ is a finite $D$-group if:

(i) $\operatorname{dim}_{K} K[G]<\infty$,

(ii) $X(G)=\{a \in K[G] \mid \Delta(a)=a \otimes a\}$

is a $K$-linear basis for $K[G]$, where $\Delta: K[G] \rightarrow K[G] \otimes K[G]$ is the comorphism induced from the multiplication morphism $m: G \times G \rightarrow G$.

Note. $X(G)$ is always a group. It is the group of characters of $G . K[G]$ is thus isomorphic to the group algebra of $X(G)$ over $K$.

2.1 Lemma. Let $X$ be an affine variety defined over $K$. Then there is a canonical one-to-one correspondence between actions of the D-group $G$ on $X$, and direct sum decompositions $K[X]=\bigoplus_{\alpha \in X(G)} K[X]_{\alpha}$ such that $K[X]_{\alpha} \cdot K[X]_{\beta} \subseteq K[X]_{\alpha+\beta}$ for all $\alpha, \beta \in X(G)$. 
Proof. Let $R=K[X]$. Given $R=\oplus R_{\alpha}$, define $\mu: R \rightarrow R \otimes K[G]$ by letting $\mu(x)=x \otimes \alpha$ if $x \in R_{\alpha}$. Plainly, this is the comorphism of an action $G \times X \rightarrow X$.

Conversely, given $\varphi: G \times X \rightarrow X$ we have $\varphi^{*}: R \rightarrow R \otimes K[G]$ which determines on $R$ the structure of a $K[G]$-comodule (i.e. $\varphi^{*}$ is coassociative and $(1 \otimes \varepsilon)^{\circ} \varphi^{*}=1$, where $\varepsilon$ is the augmentation on $K[G])$. One checks, using these two facts, that if $\varphi^{*}(a)=\sum_{\alpha \in X(G)} a_{\alpha} \otimes \alpha$ then $a=\sum_{\alpha \in X(G)} a_{\alpha}$ and $\left(a_{\alpha}\right)_{\beta}=a_{\alpha}$ if $\alpha=\beta$, and 0 otherwise. Thus, $R=\oplus_{\alpha \in X(G)} R_{\alpha}$ where $R_{\alpha}=\left\{a \in R \mid a=a_{\alpha}\right\}$. Q.E.D.

The remainder of this section is devoted to the task of sharpening some known results (see [11]) about Cohen-Macaulay rings and finite $D$-group actions. I have assumed throughout that all rings are finitely generated $K$-algebras and that $K$ is an algebraically closed field.

Let $X$ be an affine variety defined over $K$, and let $G$ be a finite $D$-group scheme such that $\varphi: G \times X \rightarrow X$ is an action of $G$ on $X$. For example, if $X$ is an algebraic group and $G$ is a closed finite $D$-subgroup scheme, then $G \times X \rightarrow X,(g, x) \rightarrow g \cdot x$, is an action of $G$ on $X$. Let $A$ be the coordinate ring of $X$.

2.2 Lemma. Let $X, A, \varphi$ be as above and let $A_{0}=\left\{x \in A \mid \varphi^{*}(x)=x \otimes 1\right\}$. Then:

(i) $A_{0}$ is a subalgebra of $A$.

(ii) The inclusion $A_{0} \rightarrow A$ is an integral extension.

(iii) $A_{\alpha}$ is an $A_{0}$-module for all $\alpha \in X(G)$.

(iv) If $A$ is a normal domain, so is $A_{0}$.

(v) $A_{0}$ is a finitely generated $K$-algebra.

Proof. (i)-(iii) are straightforward. For (iv) let $K$ and $L$ be the quotient fields of $A_{0}$ and $A$, respectively. Since $A$ is normal it suffices to prove that $A_{0}=A \cap K$. But $A \cap K$ is an $X(G)$-graded subspace of $A$ and $K=L_{0}$. Thus, $A \cap K=A \cap L_{0} \subseteq A_{0}$. (v) follows directly from (ii) and [1, Chapter $5,1.9]$. Q.E.D.

2.3 Proposition. Suppose $G \times X \rightarrow X$ is an action of the finite D-group $G$ on the normal affine variety $X$. If $X / G=\operatorname{Spec}\left(A_{0}\right)$ is smooth, then $X$ is Cohen-Macaulay.

Proof. By assumption $A$ is a normal domain and $A_{0} \rightarrow A$ is a regular subalgebra. By (2.2), $A$ is a finite $A_{0}$-module, and then by [1, Chapter 7, 4.8] it is actually reflexive. Since $A$ splits up as $\bigoplus_{\alpha \in X(G)} A_{\alpha}$, each summand $A_{\alpha}$ is reflexive. Consider now any nonzero $A_{\alpha}$, and choose $0 \neq x$ in it. Suppose $\alpha$ has order $n$ in $X(G)$. Then the map $y \rightarrow x^{n-1} \cdot y$ is injective and sends $A_{\alpha}$ into $A_{0}$. The image is an ideal that is reflexive and hence is divisorial [1, Chapter 7, 4.2]. But by assumption $A_{0}$ is regular, and hence all its divisorial ideals are projective [1, Chapter 7, 4.7]. Thus, each $A_{\alpha}$ is projective and so $A$ is projective. In particular, $A$ is flat over $A_{0}$. This implies that $A$ is Cohen-Macaulay [12, Chapter 4, D]. Q.E.D.

The following well-known result is also needed in the proof of the main theorem (3.2).

2.4 Proposition. Suppose $X$ is an irreducible, affine, Cohen-Macaulay variety and $G \times X \rightarrow X$ is an action of the finite D-group on $X$. Then $X / G$ is Cohen-Macaulay. 
Proof. Let $A=K[X]$ and $A_{0}=K[X / G] . A$ is Cohen-Macaulay as an $A$-module and thus, as an $A_{0}$-module. But $A$ splits up as $A_{0} \oplus \sum_{\alpha \in X((i) \backslash(0)} A_{\alpha}$. Thus, each summand, in particular $A_{0}$, is a Cohen-Macaulay $A_{0}$-module. Q.E.D.

\section{The main results.}

3.1 Proposition. Let $G$ be one of the groups $\mathrm{Sl}_{2}(K) \times K^{*}, \mathrm{Gl}_{2}(K)$ or $\mathrm{PGl}_{2}(K) \times$ $K^{*}$, and suppose $E$ is an irreducible, normal, algebraic monoid with 0 and unit group $G$. Then there exists a commutative diagram of algebraic monoids with $R=\operatorname{End}_{K}\left(K^{2}\right)$ :

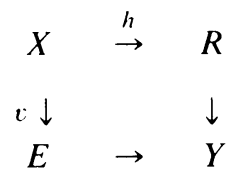

Furthermore,

(i) all morphisms are finite and dominant,

(ii) the kernel of each morphism is a finite D-group.

The proof of 3.1 is recorded in [9]. The main point is to construct the desired morphisms $v$ and $h$, first on the level of maximal $D$-submonoids, and then globally using the "big cell" $[9, \S 3] U \subseteq E$.

3.2 THEOREM. Let $E$ be an irreducible, normal, algebraic monoid with unit group $G=\mathrm{Sl}_{2}(K) \times K^{*}, \mathrm{Gl}_{2}(K)$ or $\mathrm{PGl}_{2}(K) \times K^{*}$. Then $E$ is Cohen-Macaulay.

Proof. Case 1. E has a 0.

We have, from 3.1 , the following commutative diagram with $R=\operatorname{End}_{K}\left(K^{2}\right)$ :

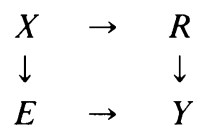

All morphisms have finite $D$-group kernels and $R$ is a smooth variety. By $2.3, X$ is Cohen-Macaulay and thus, by $2.4, E$ is Cohen-Macaulay.

Case 2. $E$ does not have a 0 .

We may assume, since groups are smooth, that $G \subsetneq E$. By [3, Corollary 1.4], $E$ has an idempotent $e \neq 1$ and from [5, Corollary 2], it follows that $e \in \bar{T}$, the closure in $E$ of some maximal torus $T$ of $G$. Let $e_{0} \in \bar{T}$ be the minimal idempotent. Then $e_{0}$ is fixed by the Weyl group $W$, so by [7, Theorem 2.3], $e_{0}$ is in the closure of the identity component $S$ of $Z G$, the center of $G$. Thus, $\operatorname{dim}\left(e_{0} T\right)>0$, since otherwise $e_{0} T=$ $\left\{e_{0}\right\}$ for all tori ( $e_{0}$ is central and all maximal tori are conjugate), contradicting the fact that $E$ does not have a 0 . Hence $I(\bar{T})=\left\{1, e_{0}\right\}$ [4, Theorem 3.7]. Let $G^{\prime}=(G$, $G)$. As $E$ is regular [10], the map $m: G^{\prime} \times \bar{S} \rightarrow E, m(x, y)=x \cdot y$, is onto, and since $G^{\prime}$ is a simple algebraic group, $m$ is finite-to-one. Thus, $E=G \cup G \cdot e ; \operatorname{dim} G=4$, $\operatorname{dim}(G \cdot e)=\operatorname{dim}\left(G^{\prime}\right)=3$. But since $E$ is normal, the singular locus has codimension larger than two. Hence, by homogeneity of $E \backslash G=G \cdot e, E$ is actually nonsingular in this case. Q.E.D. 


\section{REFERENCES}

1. N. Bourbaki, Commutative algebra, Hermann, Paris, 1972.

2. M. Hochster, Rings of invariants of tori, Cohen-Macaulay rings generated by monomials, and polytopes, Ann. of Math. (2) 96 (1972), 318-337.

3. M. Putcha, On linear algebraic semigroups, Trans. Amer. Math. Soc. 259 (1980), 457-469.

4. On linear algebraic semigroups. II, Trans. Amer. Math. Soc. 259 (1980), 471-491.

5 . Green's relation on a connected algebraic monoid, Linear and Multilinear Algebra 12 (1982), 205-214.

6. L__ Linear algebraic semigroups, Semigroup Forum 22 (1981), 287-309.

7.

8. L. Renner, Algebraic monoids, Thesis, University of British Columbia, Vancouver, 1982.

9. Classification of semisimple rank one monoids (to appear).

10. . Reductive monoids are von Neumann regular (to appear).

11. P. Roberts, A helian extensions of regular local rings, Proc. Amer. Math. Soc. 78 (1980), 307-310.

12. J. Serre, Algèbre locale, Lecture Notes in Math., vol. 11, Springer-Verlag, Berlin, 1966.

13. W. Waterhouse, Introduction to affine group schemes, Graduate Texts in Math., vol. 66, SpringerVerlag, Berlin, 1979.

Department of Mathematics, York University, Downsview, Ontario, Canada M3J IP3 